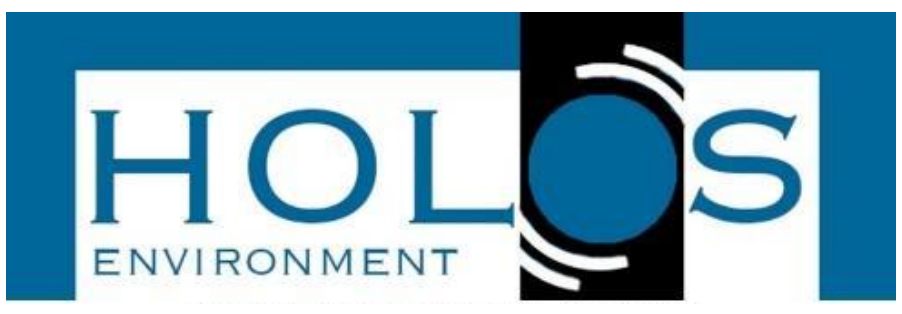

\title{
MANEJO DE MACRÓFITAS PROVENIENTE DE INFESTAÇÃO EM AMBIENTES NATURAIS COMO SUPLEMENTO PARA PRODUÇÃO DA MICROALGA DE INTERESSE ECONÔMICO Scenedesmus acuminatus
}

\section{MACROPHYTE MANAGEMENT FROM INFESTATION IN NATURAL ENVIRONMENTS AS A SUPPLEMENT FOR THE PRODUCTION OF ECONOMIC INTEREST MICROALGAE Scenedesmus acuminatus}

\author{
Frederico Pacheco Militão"; Raissa Hirle Krettle"; Fernanda Brêda-Alves ${ }^{1}$; Camilo Dias Júnior ${ }^{1}$; Erico \\ Tadao Teramoto²; Levi Pompermayer Machado²; Valéria De Oliveira Fernandes ${ }^{1}$
}

Artigo recebido em: 28/02/2020 e aceito para publicação em: 08/03/2020. DOI: http:/dx.doi.org/10.14295/holos.v20i2.12379

\begin{abstract}
Resumo: Este estudo avaliou a influência de seis meios de cultura alternativos (um a base de NPK, dois contendo extrato de Pistia stratiotes e Eichhornia crassipes enriquecido de NPK, e outros três com diferentes diluições de extrato puro de E. crassipes, (Eic 40, Eic 85, Eic 130) em comparação ao meio de cultura comercial ASM-1, no desenvolvimento da microalga Scenedesmus acuminatus. Foram avaliados taxa de crescimento $(\mathrm{K})$, densidade, volume celular, biovolume e qualidade nutricional dos meios de cultura. Os meios de culturas testados apresentaram concentração de nutrientes suficiente para o crescimento da microalga. As taxas de crescimento de ASM-1 $(0,439)$ foram similares $(p>0,05)$ a NPK, Pis + NPK e Eic + NPK. Eic 85 e Eic 130 apresentaram os valores mais baixos $(0,201 ; 0,150$, respectivamente) e similares entre si. Os volumes dos tratamentos não diferiram de ASM-1 (135,427 $\mu \mathrm{m} 3)$, somente o Eic NPK e Eic 130 apresentaram valores menores $(86,780$ e $100,401 \mu \mathrm{m} 3$, respectivamente). O maior biovolume e densidade celular foram encontrados em Eic NPK (1,273 mm3.L-1 e 1047 x 104 cél.mL-1). Os tratamentos apresentaram capacidade de nutrição muito similar ao ASM-1, podendo substitui-lo no cultivo de S. acuminatus, em especial Eic + NPK que apresentou maior densidade e biovolume. Os tratamentos com extrato puro, apesar de apresentarem resultados mais baixos, ainda assim possibilitaram o desenvolvimento da alga.
\end{abstract}

Palavras-chave: Cultivo. Microalga. Macrófitas. NPK. Meio de cultura.

Abstract: This study evaluated the influence of six alternative culture media (one of NPK, two containing Pistia stratiotes and Eichhornia crassipes extracts enriched with NPK, and three with different dilutions of pure extract of E. crassipes (Eic 40, Eic 85, Eic 130) compared to the commercial culture medium ASM-1 in the development of the microalga Scenedesmus acuminatus. Growth rate (K), density, cell volume, biovolume and nutritional quality of culture media were evaluated. The tested media had enough concentration of nutrients for the growth of microalgae. The growth rate of ASM-1 (0.439) were similar ( $p>0.05)$ to NPK, NPK + Pis and Eic + NPK. Eic 85 and Eic 130 had the lowest values $(0.201 ; 0.150$, respectively) and similar to each other. The volumes of the treatments did not differ to ASM-1 (135.427 $\mu \mathrm{m} 3)$, only Eic + NPK and Eic 130 had lower values (86.780 and $100.401 \mu \mathrm{m} 3$, respectively). The highest biovolume and cell density were found in Eic + NPK (1.273 mm3.L-1 and $1047 \times 104$ cél.mL-1). It was concluded that the treatments had very similar nutritional ability to ASM-1 and may replace it in the cultivation of $S$. acuminatus. In particular Eic + NPK with the highest density and biovolume. The treatments with pure extract, despite having lower results, still allowed the development of algae.

Keywords: Culturing. Microalgae. Macrophytes. NPK. Culture medium.

\footnotetext{
${ }^{1}$ Universidade Federal do Espírito Santo (UFES), Vitória, ES. E-mail: (fredericomilitao@gmail.com, raissahk@hotmail.com, f.bredaalves@hotmail.com, camilo.junior@terra.com.br, valeriaufes@yahoo.com.br)

2 Universidade Estadual Paulista "Julio de Mesquita Filho" (UNESP), Registro - SP. E-mail: (erico.teramoto@unesp.br, levi.p.machado@unesp.br)
} 


\section{INTRODUÇÃO}

As microalgas vêm sendo isoladas do ambiente e cultivadas para diferentes finalidades, que vão desde estudos ecológicos ou ambientais e mais recentemente para aplicações biotecnológicas e comerciais (LOURENÇO, 2006). Essa ferramenta possibilita compreender o comportamento ecofisiológico das espécies em resposta a condições ambientais. Por meio do cultivo é possível avaliar plasticidade e a resposta das algas a diferentes variáveis ambientais, o ciclo de vida e auxiliar na sistemática biológica dos grupos. Além disso, a biomassa das algas pode ter aplicações de interesse econômico, como a obtenção de biomoléculas, uso como suplemento alimentar humano e animal, ou fonte de biocombustíveis (LOURENÇO, 2006).

O gênero Scenedesmus é atualmente muito visado para o cultivo devido ao seu crescimento rápido, fácil adaptação às variações ambientais (TRAINOR, 1998) e grande valor nutritivo com alta produção de proteína e ácidos graxos (MILITÃO et al., 2019). O seu cultivo é voltado principalmente para a aquicultura (DERNER et al., 2006), para a alimentação humana como suplemento alimentar e como potencial matéria prima para produção de biodiesel (PRABAKARAN; RAVINDRAN, 2012).

Apesar do grande potencial, o cultivo de microalgas ainda apresenta custo elevado, sendo um dos principais, o custo dos reagentes químicos utilizados na preparação dos meios de cultura comerciais (SIPAÚBA-TAVARES e PEREIRA, 2008; FERRELL e SARISKYREED, 2010; KRUSE e HANKAMER, 2010).

Vários estudos vêm sendo realizados no intuito de avaliar o crescimento dessas microalgas cultivadas em meios de cultura alternativos, que mesmo sendo baratos e facilmente obtidos, são ricos em nutrientes necessários para o crescimento e desenvolvimento desses organismos. Os meios alternativos são produzidos através de diferentes fontes orgânicas e inorgânicas, como efluentes municipais, industriais, de agricultura, biodigestores, extrato de solo, dentre outras (DALRYMPLE et al., 2013; ALMEIDA, 2007; PIÑA et al., 2007; FIORESI e SIPAÚBA-TAVARES, 2008). A biomassa de macrófitas aquáticas, por sua riqueza de micro e macronutrientes, também foram sugeridas como fertilizantes orgânicos de solo, de piscicultura e como meio de cultura para microalgas (SAMPAIO e OLIVEIRA, 2005; SIPAÚBA-TAVARES e BRAGA, 2007).

As macrófitas aquáticas flutuantes Eichhornia crassipes e Pistia stratiotes são amplamente distribuídas nos ambientes aquáticos dulcícolas, principalmente em ambientes tropicais, e são consideradas pragas devido ao seu crescimento acelerado e fácil adaptação 
(CAMARGO et al., 2003; MARTINS et al., 2003). A proliferação excessiva dessas macrófitas em ambientes aquáticos naturais desencadeiam uma série de problemas ambientais e econômicos como a redução da biodiversidade local, proliferação de insetos transmissores de doenças, obstáculo para o transporte aquático, prejudicando a pesca e o abastecimento de água (NDIMELE et al., 2011; LIMA et al., 2018).

Em contrapartida, a alta taxa de crescimento, ampla distribuição e facilidade de colonizar novos ambientes, associados à grande capacidade de estocarem nutrientes, tornam as macrófitas atrativas do ponto de vista econômico (HENRY-SILVA e CAMARGO, 2006). Neste contexto, estudos vêm sendo realizados afim de avaliar a aplicação de macrófitas na absorção de metias pesados (BARTMEYER et al., 2019) e no tratamento de águas residuárias (LEMES et al., 2008), no entanto, não existe ainda tecnologia para descarte adequado ou utilização da biomassa final gerada (SILVA et al., 2019).

A utilização de meio de cultivo contendo extrato de macrófitas aquáticas, como Pistia stratiotes e Eichhornia crassipes, que infestam ambientes aquáticos eutrofizados, complementados ou não com a utilização de fertilizante NPK (SIPAÚBA-TAVARES e ROCHA, 1993), pode servir de importante fonte alternativa em virtude de suas concentrações elevadas de nitrogênio, fósforo e potássio (SIPAÚBA-TAVARES e BRAGA, 2007) além de outros nutrientes como magnésio, enxofre, manganês, cobre, zinco, ferro e cálcio (HENRYSILVA e CAMARGO, 2006), essenciais ao desenvolvimento das microalgas, além de contribuir para a reutilização de resíduos naturais, dando uma destinação sustentável a biomassa dessas plantas aquáticas.

Porém, assim como outros extratos orgânicos como efluentes e biodigestores, o extrato de macrófita bruto apresenta alta turbidez, devido à presença de pigmentos e substâncias orgânicas dissolvidas. Essa coloração pode trazer prejuízo ao crescimento das microalgas pela atenuação da passagem de luz, diminuindo assim a fotossíntese (TAHER, 2013).

Devido à importância econômica do cultivo de microalgas é necessário estudar maneiras ambientalmente viáveis e que proporcionem a redução do custo de cultivo. Tendo em vista que os meios de cultura representam um dos itens de maior gasto para o cultivo em larga escala, o uso de meios alternativos e de fácil aquisição, como extrato de macrófitas e NPK, pode auxiliar a viabilizar o processo produtivo dos cultivos. Desta forma no presente trabalho avaliamos a utilização de macrófitas coletadas em ambientes naturais, que apresentavam problemas de infestação, como fonte de nutrientes para o cultivo da microalga Scenedesmus acuminatus. Para tanto foram realizados 5 tratamentos com diferentes fontes 
de nutrientes adicionados em água destilada utilizando tanto extratos de macrófitas somados aos macronutrientes NPK, diluições dos extratos de macrófitas puro e os contendo apenas os macronutrientes NPK puro e a solução de nutrientes ASM 1, utilizado comercialmente para cultivos de microalgas.

\section{METODOLOGIA}

\subsection{Cepa usada}

A cepa de Scenedesmus acuminatus (L027A) foi obtida no Laboratório de Taxonomia e Ecologia de Algas Continentais (LATEAC) pertencente ao Departamento de Ciências Biológicas da UFES.

\subsection{Preparação dos extratos das macrófitas aquáticas}

As macrófitas aquáticas selecionadas foram Eichhornia crassipes (Mart.) Solms (aguapé) e Pistia stratiotes L. (alface d'água). Os exemplares foram coletados na lagoa Juara (Serra -ES), o ambiente é usado para múltiplas atividades e apresenta problemas ecológicos devido ao elevado crescimento de macrófitas. As biomassas foram lavadas e secas ao sol por $24 \mathrm{~h}$ e após colocadas em estufas à $60^{\circ} \mathrm{C}$ por mais $24 \mathrm{~h}$. Para a preparação do extrato $82 \mathrm{~g}$ de $E$. crassipes e $164 \mathrm{~g}$ de $P$. stratiotes secas foram moídas e posteriormente fervidas em 1,8L e 2,2L de água destilada, respectivamente. Cada extrato ainda quente foi filtrado e autoclavado a $120^{\circ} \mathrm{C}$, durante 20 minutos.

\subsection{Delineamento experimental}

Foram testados seis tratamentos, além do controle, todos realizados em triplicata. Os experimentos foram feitos em erlenmeyers de $500 \mathrm{~mL}$, com volume final de $316 \mathrm{~mL}$. Como controle usou-se o meio comercial ASM-1, turbidez 0 NTU; tratamento NPK (20:05:20), com $316 \mathrm{~mL}$ de água destilada e $0,11 \mathrm{~g}$ de NPK, turbidez 0 NTU; Pis+NPK, com $16 \mathrm{~mL}$ de extrato de $P$. stratiotes adicionados a $300 \mathrm{ml}$ de água destilada e 0,11 g de NPK, turbidez 5,78 NTU; Eic+NPK, com $16 \mathrm{~mL}$ de extrato de $E$. crassipes adicionados a $300 \mathrm{~mL}$ de água destilada e $0,11 \mathrm{~g}$ de NPK, turbidez 18,3 NTU; Eic40, com $40 \mathrm{~mL}$ de extrato de E. crassipes 
adicionados a $276 \mathrm{~mL}$ de água destilada, turbidez 50,33; Eic85, com $85 \mathrm{~mL}$ de extrato de $E$. crassipes adicionados a $231 \mathrm{~mL}$ de água destilada, turbidez 126,9 NTU e o tratamento Eic130, com $130 \mathrm{~mL}$ de extrato de E. crassipes adicionados a $186 \mathrm{~mL}$ de água destilada com turbidez de 209,98 NTU. A turbidez de cada tratamento foi determinada no início do experimento.

$\mathrm{O} \mathrm{pH}$ das unidades experimentais foi 7,0. $\mathrm{O}$ experimento foi realizado na sala de cultivo do LATEAC, com iluminação fornecida por duas lâmpadas do tipo daylight de $40 \mathrm{~W}$, por bancada, temperatura de $25 \pm 2^{\circ} \mathrm{C}$ e fotoperíodo de $24 \mathrm{~h}$. Inóculo inicial (dia 0 ) de $30,7 \mathrm{x}$ $10^{4}$ células. $\mathrm{mL}^{-1}$.

\subsection{Análise dos nutrientes}

Amostras dos extratos de $P$. stratiotes e E. crassipes, e de meio com NPK puro foram enviados ao laboratório CQA (Centro de Qualidade Analítica LTDA) para a determinação, nas três amostras, da concentração ortofosfato, pelo método EPA 3050B / 1996, nitrogênio amoniacal pelo método SM4500, nitrito, nitrato pelo FQ-0279-02, e nos extratos de macrófitas foram quantificados nas três amostras e ferro, manganês, cobre, zinco, boro pelo método EPA 6010B/1996 e enxofre pelo EPA SW 846/1994.

\subsection{Avaliação do crescimento de Scenedesmus acuminatus}

Para a composição da curva de crescimento, foram retiradas alíquotas de $1 \mathrm{~mL}$ a cada dois dias, durante vinte e quatro dias. A quantificação dos indivíduos (ind. $/ \mathrm{mL}$ ) foi feita por meio de câmara de Neubauer. A taxa de crescimento (K) foi calculada utilizando a fase exponencial da curva de crescimento, representada pelo número de divisões celulares da população por dia, de acordo com Stein (1973).

\subsection{Biovolume}

Para análise do volume celular foram realizadas medições da altura e do diâmetro $(\mu \mathrm{m})$ de 20 células de $S$. acuminatus. A figura geométrica que melhor representou a forma da alga usada foi a de dois cones acoplados. O volume celular médio $\left(\mu \mathrm{m}^{3}\right)$ foi calculado utilizando a fórmula 1. 
Sendo, $d$ = diâmetro, $h$ = altura. $\mathrm{O}$ biovolume $\left(\mathrm{mm}^{3} \cdot \mathrm{L}^{-1}\right)$ foi calculado multiplicando o volume celular médio $\left(\mu \mathrm{m}^{3}\right)$ pelo $\mathrm{n}^{\circ}$ de células contadas (cél. $\mathrm{mL}^{-1}$ ) no $20^{\circ}$ dia, no qual os experimentos já haviam atingido a fase estacionária (fórmula 2).

Biovolume $\left(\mathrm{mm}^{3} \cdot \mathrm{L}^{-1}\right)=\mathrm{n}^{\circ}$ de células. $\mathrm{mL}^{-1}$ * volume celular $\left(\mu \mathrm{m}^{3}\right)$ * $10^{-9}$

\subsection{Tratamento estatístico}

Os dados foram submetidos a teste de normalidade (Kolmogorov-Smirnov), sendo os dados normais comparados por ANOVA seguido de teste de Tukey e os não normais pelo teste Kruskal-Wallis seguido de teste de student-Newman-Keuls, utilizando o programa BIOESTAT 5.0. Foram considerados dados com variação significativa para o nível de confiança de $95 \%(p<0,05)$. As análises descritivas também foram feitas utilizando o mesmo programa.

\section{RESULTADOS}

A composição química do meio ASM1, utilizado como controle, está descrita na tabela 1. Os nutrientes disponíveis no volume final dos tratamentos estão descritos na tabela 2, a seguir.

Tabela 1 - Composição química do meio ASM-1.

\begin{tabular}{|c|c|}
\hline Componente & $\mathrm{mg} / 316 \mathrm{~mL}$ \\
\hline $\mathrm{NaNO}_{3}$ & 53,72 \\
\hline $\mathrm{MgSO}_{4} \cdot 7 \mathrm{H}_{2} \mathrm{O}$ & 15,484 \\
\hline $\mathrm{MgCl}_{2} \cdot 6 \mathrm{H}_{2} \mathrm{O}$ & 12,956 \\
\hline $\mathrm{CaCl}_{2} \cdot 2 \mathrm{H}_{2} \mathrm{O}$ & 9,164 \\
\hline $\mathrm{KH}_{2} \mathrm{PO}_{4}$ & 5,4984 \\
\hline $\mathrm{Na}_{2} \mathrm{HPO}_{4}$ & 4,46192 \\
\hline $\mathrm{H}_{3} \mathrm{BO}_{3}$ & 0,78368 \\
\hline $\mathrm{MnCl}_{2} \cdot 4 \mathrm{H}_{2} \mathrm{O}$ & 0,43924 \\
\hline $\mathrm{FeCl}_{3} \cdot 6 \mathrm{H}_{2} \mathrm{O}$ & 0,34128 \\
\hline $\mathrm{ZnCl}_{2}$ & 0,10586 \\
\hline $\mathrm{CoCl}_{2} \cdot 6 \mathrm{H}_{2} \mathrm{O}$ & 0,006 \\
\hline $\mathrm{CuCl}_{2} \cdot 2 \mathrm{H}_{2} \mathrm{O}$ & 0,0004424 \\
\hline $\mathrm{EDTA} \cdot \mathrm{Na}_{2}$ & 2,35104 \\
\hline
\end{tabular}


Tabela 2 - Composição nutricional dos tratamentos

\begin{tabular}{|c|c|c|c|c|c|c|}
\hline Componente & $\begin{array}{l}\text { Pis+NPK } \\
\text { (mg/316mL) }\end{array}$ & $\begin{array}{l}\text { Eic+NPK } \\
(\mathrm{mg} / 316 \mathrm{~mL})\end{array}$ & $\begin{array}{l}\text { NPK } \\
\text { (mg/316mL) }\end{array}$ & $\begin{array}{l}\text { Eic } 40 \\
(\mathrm{mg} / 316 \mathrm{~mL})\end{array}$ & $\begin{array}{l}\text { Eic } 85 \\
(\mathrm{mg} / 316 \mathrm{~mL})\end{array}$ & $\begin{array}{l}\text { Eic130 } \\
\text { (mg/316mL) }\end{array}$ \\
\hline Ortofosfato & 12,15 & 12,01 & 8,52 & 8,728 & 18,547 & 28,37 \\
\hline $\mathrm{N}$-amoniacal & 109,18 & 108,88 & 96,38 & 31,24 & 66,38 & 101,53 \\
\hline $\mathrm{N}$-nitrito & 2,55 & 2,53 & 2,41 & 0,3 & 0,64 & 0,98 \\
\hline N-nitrato & 0,315 & 0,315 & 0,30 & 0,04 & 0,08 & 0,12 \\
\hline Ferro & 0,059 & 0,063 & - & 0,158 & 0,336 & 0,51 \\
\hline Manganês & 0,234 & 0,39 & - & 0,98 & 2,08 & 3,19 \\
\hline Cobre & ${ }^{* *}<\mathrm{LQ}$ & ${ }^{* *}<\mathrm{LQ}$ & - & ${ }^{* *}<\mathrm{LQ}$ & ${ }^{* *}<\mathrm{LQ}$ & ${ }^{* *}<\mathrm{LQ}$ \\
\hline Zinco & ${ }^{* *}<\mathrm{LQ}$ & ${ }^{* *}<\mathrm{LQ}$ & - & ${ }^{* *}<\mathrm{LQ}$ & ${ }^{* *}<\mathrm{LQ}$ & ${ }^{* *}<\mathrm{LQ}$ \\
\hline Boro & ${ }^{* *}<\mathrm{LQ}$ & ${ }^{* *}<\mathrm{LQ}$ & - & ${ }^{* *}<\mathrm{LQ}$ & ${ }^{* *}<\mathrm{LQ}$ & ${ }^{* *}<\mathrm{LQ}$ \\
\hline Enxofre & 8,2 & 1,92 & - & 4,8 & 10,2 & 15,6 \\
\hline
\end{tabular}

${ }^{* *}<\mathrm{LQ}$ : Menor que o limite de quantificação

As curvas de crescimento dos seis tratamentos e do controle estão demonstradas na figura 1. Somente Eic 85 e Eic 130 apresentaram fase de adaptação, no primeiro essa fase durou até o $4^{\circ}$ dia e no segundo até o dia 16, iniciando sua fase log a partir daí até o fim do experimento. Todos os outros tratamentos e o controle apresentaram fase exponencial a partir do primeiro dia indo até o 8o dia no controle (ASM1) e NPK, até o dia 12 em Eic NPK, até o dia 8 para Pis NPK e Eic 40, e o Eic 85 teve a fase exponencial identificada do dia 4 ao 18.

O tratamento Eic NPK foi o que atingiu maiores densidades celulares, $1047 \times 10^{4}$ cél. $\mathrm{mL}^{-1}$ no $24^{\circ}$ dia, seguido do ASM1 com 826 × $10^{4}$ cél. $\mathrm{mL}^{-1}$ no $22^{\circ}$ dia, NPK com $782 \times 10^{4}$ cél. $\mathrm{mL}^{-1}$ no $24^{\circ}$ dia, Pis NPK, $426 \times 10^{4}$ cél. $\mathrm{mL}^{-1}$, no $22^{\circ}$ dia e Eic $40,414 \times 10^{4}$ cél. $\mathrm{mL}^{-1}$, no

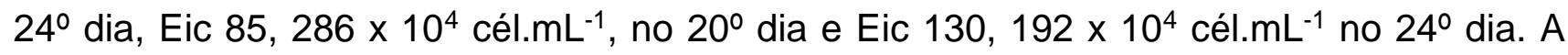
queda dos crescimentos apresentada no dia 14 foi caracterizada por uma variação metodológica comum aos tratamentos, portanto não deve ser considerada na avaliação. As médias das taxas de crescimento, biovolume e volume das células estão expostas na tabela 3.

Figura 1- Curvas de crescimento de Scenedesmus acuminatus em cada tratamento

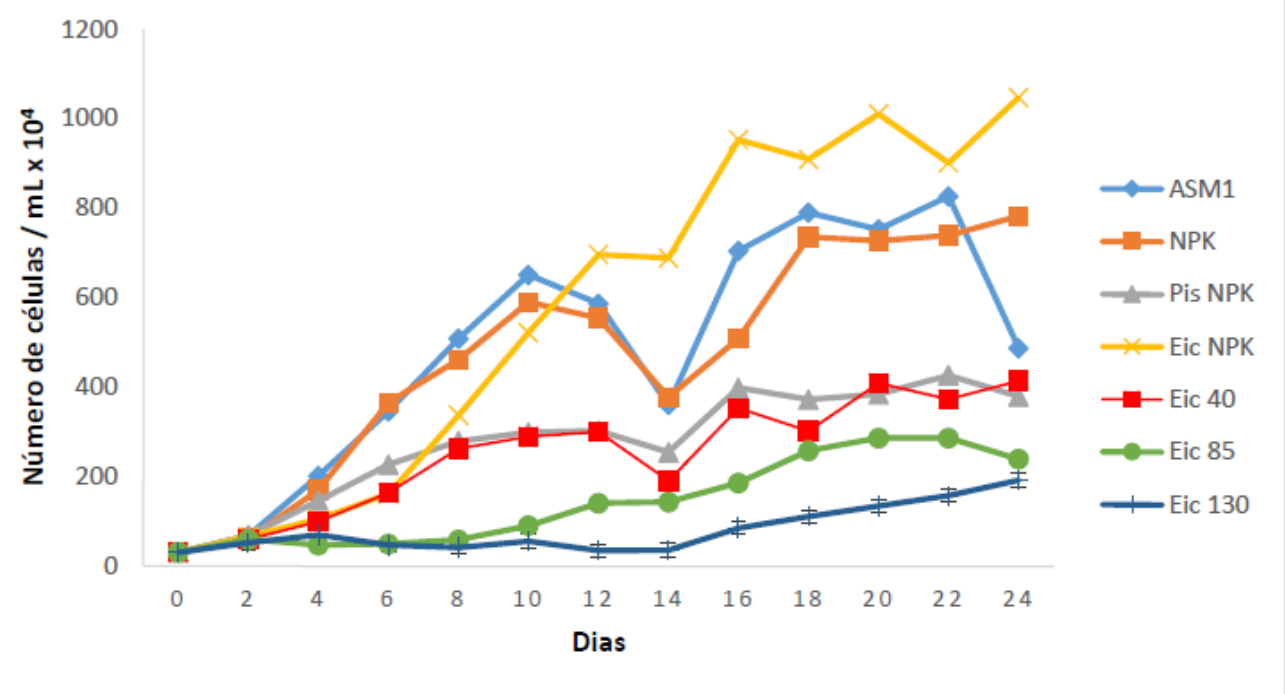


Tabela 3 - Valores médios de K (taxa de crescimento), biovolume e volume celular de Scenedesmus acuminatus nos tratamentos e controle

\begin{tabular}{lccc}
\hline Tratamentos & $\mathbf{K}$ (dia) & $\begin{array}{c}\text { Biovolume } \\
\left(\mathbf{m m}^{3} . \mathbf{L}^{-1}\right)\end{array}$ & Volume $\left(\boldsymbol{\mu \mathbf { m } ^ { 3 } )}\right.$ \\
\hline ASM1 & $0,439 \pm 0,023 \mathrm{a}$ & $1,052 \pm 0,063 \mathrm{a}$ & $135,427 \pm 13,8 \mathrm{ab}$ \\
NPK & $0,425 \pm 0,026 \mathrm{a}$ & $0,964 \pm 0,045 \mathrm{ab}$ & $152,257 \pm 14,2 \mathrm{ab}$ \\
Pis NPK & $0,395 \pm 0,036 \mathrm{ab}$ & $1,092 \pm 0,413 \mathrm{a}$ & $336,996 \pm 146,6 \mathrm{a}$ \\
Eic NPK & $0,408 \pm 0,019 \mathrm{a}$ & $1,273 \pm 0,665 \mathrm{a}$ & $86,780 \pm 11,1 \mathrm{~b}$ \\
Eic 40 & $0,323 \pm 0,004 \mathrm{~b}$ & $0,928 \pm 0,178 \mathrm{ab}$ & $220,586 \pm 61,1 \mathrm{a}$ \\
Eic 85 & $0,201 \pm 0,040 \mathrm{C}$ & $0,515 \pm 0,264 \mathrm{~b}$ & $155,871 \pm 74,8 \mathrm{ab}$ \\
Eic 130 & $0,150 \pm 0,042 \mathrm{c}$ & $0,268 \pm 0,193 \mathrm{C}$ & $100,401 \pm 25,3 \mathrm{~b}$ \\
\hline * Média \pm DV $(\mathrm{n}=3)$. Letras diferentes representam variação significativa $\mathrm{p}<0,05$.
\end{tabular}

Os valores de K para ASM1, NPK, Eic NPK e Pis NPK não apresentaram diferença significativa entre si, sendo este último semelhante estatisticamente também ao Eic 40. Os menores valores de K obtidos foram observados em Eic 85 e Eic 130, não apresentando diferença significativa entre si. O volume de S. acuminatus em ASM 1, NPK, Eic 40, Eic 85 e Eic 130 não apresentaram diferença significativa entre si. Os maiores valores foram encontrados nos tratamentos Pis NPK, Eic 40 e Eic 85, sem diferença significativa entre eles. O biovolume de ASM1, NPK, Pis NPK, Eic NPK e Eic 40 apresentaram valores estatisticamente semelhantes, sendo Pis NPK, Eic NPK e Eic 40 semelhantes também ao Eic 85 . Eic 130 apresentou o valor mais baixo e estatisticamente semelhante somente ao Eic 85.

\section{DISCUSSÃO}

Os seis tratamentos apresentaram valores de nitrato menores do que o controle ASM1, porém, compensaram esse valor com nitrogênio amoniacal, não presente no meio comercial. O nitrogênio amoniacal tem vantagens em relação ao nitrato pelo fato de ser mais facilmente absorvido pelas algas uma vez que não precisa ser reduzido a nitrito e a amônio antes de ser incorporado (YIN et al., 1998), gerando economia energética.

O fósforo por sua vez, foi encontrado em quantidades aproximadamente iguais em quase todos os tratamentos em relação ao ASM1, exceto Eic 85 que apresentou quase $o$ dobro e Eic 130, quase o triplo. Esse nutriente é requerido em pequenas quantidades pelas algas. Quando em grandes quantidades, podem causar inibição como documentado em Baumgartner et al. (2013). Essa inibição pode ter contribuído para menor densidade apresentada em Eic 85 e Eic 130. 
Os micronutrientes detectados nos extratos foram manganês, ferro e enxofre. Os demais micronutrientes estavam abaixo da capacidade de quantificação dos testes químicos usados, porém não significa que eles não estavam presentes.

Lima et al. (2003) comparando massa seca de Eichhornia e Pistia não encontraram diferenças significativas entre os macro e micro nutrientes das duas espécies, assim como a quantificação nutricional dos extratos das duas macrófitas do presente trabalho não diferiu grandemente entre si.

A quantificação dos nutrientes do extrato de Eichhornia + NPK em Sipaúba-Tavares et al. (2009) apresentaram valores muito menores dos encontrados no presente trabalho (Tabela 4). Além disso, o valor de fosfato encontrado por aqueles autores foi superior aos valores de nitrogênio, o que é atípico, visto que compostos nitrogenados, normalmente, são mais abundantes do que fósforo em NPK 20:05:20 e em macrófitas (HENRY-SILVA e CAMARGO, 2006; HENRY-SILVA e CAMARGO, 2002; SIPAÚBA-TAVARES e BRAGA, 2007).

Tabela 4 - Comparação dos nutrientes presentes nos extratos Eichhornia + NPK dos dois trabalhos

\begin{tabular}{lcc} 
& Sipaúba-Tavares et al. (2009) & Presente estudo \\
\hline Amônia $(\mathrm{mg} / 316 \mathrm{~mL})$ & 0,028 & 108,88 \\
Nitrito $(\mathrm{mg} / 316 \mathrm{~mL})$ & 0,00021 & 2,53 \\
Nitrato $(\mathrm{mg} / 316 \mathrm{~mL})$ & 0,045 & 0,315 \\
Ortofosfato $(\mathrm{mg} / 316 \mathrm{~mL})$ & 0,098 & 12,01 \\
\hline
\end{tabular}

Fonte: Sipaúba-Tavares et al. (2009) e dados do próprio autor

Essas variações podem ter ocorrido por alguma diferença metodológica na confecção do extrato, ou pelo fato do NPK ser de marca diferente e as Eichhornias terem sido coletadas de lugares distintos, tendo, portanto, variações nutricionais.

Assim como o presente trabalho, Henry-Silva e Camargo (2006) também registraram valores maiores de manganês e ferro na massa seca de Pistia e Eichhornia, em relação aos outros micronutrientes. Igualmente Sipaúba-Tavares e Braga (2007) também encontraram essa proporção em extrato de Eichhornia.

Como estas macrófitas aquáticas são filtradoras do meio em que vivem, absorvendo os nutrientes do meio e incorporando em sua biomassa, esses valores nutricionais provavelmente são passíveis de mudanças. Dessa forma, os nutrientes encontrados nos extratos, possivelmente, refletem os ambientes em que vivem. Em estudo realizado na lagoa Juara, Almeida e Fernandes (2013) registraram altas concentrações de ortofosfato, fósforo total, nitrogênio total e nitrogênio amoniacal, classificando esse ambiente como eutrófico. Duarte (2014) registrou concentração de Fe de 1,237 a 2,614 mg/L, superior ao valor máximo 
estabelecido pelo CONAMA (0,3 mg/L) para lagoas e concentrações de manganês de 0,04 a 0,056 mg/L. Isso pode ter influenciado nas concentrações registradas no extrato das duas macrófitas.

O alto índice de enxofre encontrado nos extratos pode ser compreendido pela abundância desse elemento em matéria orgânica principalmente na composição de aminoácidos (LOURENÇO, 2006).

De modo geral, os compostos encontrados nos extratos de Pistia e Eichhornia apresentaram variedade e concentrações suficientes para o crescimento de Scenedesmus acuminatus.

Porém, o tratamento Pistia + NPK apresentou valores de crescimento menores do que NPK puro, sugerindo que a adição do extrato prejudicou um pouco o crescimento da alga. Esse fato pode ser explicado por algum possível composto alelopático produzido pela macrófita, o qual acabou sendo transferido para o extrato. Já foi documentado ação inibitória de metabólitos secundários produzidos por Pistia stratiotes sobre algas (GRECA et al., 1999).

Os valores de $\mathrm{K}$ obtidos no presente trabalho para Eic + NPK e controle foram parecidos aos registrados por Sipaúba-Tavares et al. (2009), cultivando Ankistrodesmus gracilis, $(0,36$ e 0,4 , respectivamente), entretanto o meio comercial usado por eles foi o $\mathrm{CHU}$ 12.

Assim como no presente estudo, onde a densidade de Eic + NPK alcançou densidade de $1047 \times 10^{4}$ cél. $\mathrm{mL}^{-1}$, superior ao controle com $826 \times 10^{4}$ cél. $\mathrm{mL}^{-1}$, a densidade máxima alcançada pelo extrato enriquecido de Sipaúba-Tavares et al. (2009) $\left(525,02 \times 10^{5}\right.$ células. $\left.\mathrm{mL}^{-1}\right)$ também foi maior do que o meio convencional $\left(447,93 \times 10^{5}\right.$ células. $\left.\mathrm{mL}^{-1}\right)$, demonstrando o potencial do extrato dessa macrófita acrescido de NPK em substituir os meios de cultura comerciais.

Sipaúba-Tavares et al. (2011) também usaram NPK e EIC + NPK, porém em três diferentes recipientes (recipiente de fibra de vidro, garrafão e sacola plástica) para cultivar $A$. gracilis e as taxas de crescimento foram menores em todos os tratamentos, em relação ao presente estudo. Os valores de k obtidos para Eic + NPK, na ordem recipiente de fibra de vidro, garrafão e sacola plástica, foram: 0,$30 ; 0,14 ; 0,16$. Os resultados foram justificados devido à coloração escura do extrato. Apesar da diluição ter sido a mesma usada no presente trabalho, o volume dos recipientes foi de 13L, e isso pode ter intensificado a coloração final trazendo mais prejuízo em comparação ao volume usado nesta pesquisa $(316 \mathrm{~mL})$.

Os resultados de $\mathrm{K}$ para NPK, desse trabalho, na mesma ordem acima (SIPAÚBATAVARES et al., 2011) foram: 0,38; 0,24; 0,46. Somente o cultivo em sacola plástica 
apresentou valor de K próximo ao encontrado no tratamento com NPK no presente estudo, os outros recipientes apresentaram taxas de crescimento menores. Somente o meio controle usado ( $\mathrm{CHU}$ ) apresentou resultados de $\mathrm{K}$ e densidade celular próximos ao controle (ASM1): $0.40 ; 0.42 ; 0.44$ e $70.85 \times 10^{5} ; 62.92 \times 10^{5} ; 73.06 \times 10^{5}$ (recipiente de fibra de vidro, garrafão e sacola plástica, respectivamente).

Baumgartner et al. (2013), cultivando S. acuminatus em meio com NPK (20:05:20) e dois meios de cultura comerciais $\mathrm{MC}$ e $\mathrm{DM}$, apresentou resultados inferiores para $\mathrm{K}$ no meio com NPK em relação aos meios comerciais, justificando esse resultado pelo alto valor de fósforo presente nesse meio. De fato, a concentração desse componente no meio foi de 515 $\mathrm{mg} / 316 \mathrm{~mL}$ (ou 1,63 g.L-1), muito maior se comparado aos 8,52 mg / $316 \mathrm{~mL}$ encontrados no presente estudo, que obteve resultados satisfatórios do NPK em relação meio de cultura comercial. Com isso, pode-se inferir que altas doses de nutrientes, principalmente fósforo, podem ser prejudiciais ao desenvolvimento dessa espécie.

Em vários estudos as macrófitas aquáticas já demostraram possuir nutrientes suficiente em sua composição para serem usadas na fertilização de culturas. Tepe et al. (2006) usaram massa seca de três espécies de macrofitas e lodo como única fonte de nitrogênio para cultivo de Scenedesmus acuminatus. A macrófita Nymphaea, por possuir maior porcentagem de nitrogênio em sua composição (1,96\%) em relação a Typha e Myriophyllum (1,21\% e 1,53\%), apresentou cultivo com maior densidade dessa alga (158 $\mathrm{x}$

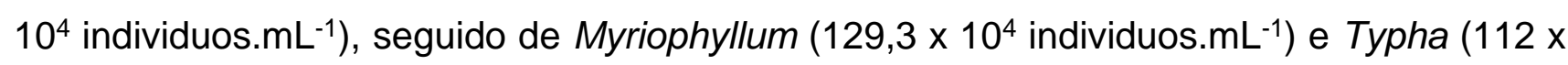
$10^{4}$ individuos. $\mathrm{mL}^{-1}$ ). Essas três densidades encontradas por Tepe et al. (2006) foram superiores às encontradas no presente trabalho para os tratamentos e o controle, porém juntamente com as macrófitas estes autores acrescentaram outros nutrientes que não foram acrescentados aqui. Variações na densidade celular de microalgas entre os trabalhos podem ocorrer devido a diferenças de metodologias e cepas utilizadas.

Ao analisar o resultado obtido nos três tratamentos com diferentes diluições do extrato puro de Eichhornia (Eic 40, 85 e 130), foi possível confirmar sua capacidade de nutrição para a alga, possibilitando seu crescimento; entretanto, a turbidez do extrato pareceu influenciar o cultivo de $S$. acuminatus. Apesar dos três tratamentos terem apresentado concentração crescente de nutrientes pela presença de maior volume de extrato $(40,85$ e $130 \mathrm{~mL})$, os valores de $\mathrm{K}$ e densidade celular máxima foram inversamente proporcionais à turbidez do meio.

Isso ocorre porque à medida que a turbidez aumenta ocorre maior dispersão e absorção da luz pelas partículas dissolvidas ou não no meio, o que impede que a penetração 
de luz ocorra favoravelmente em linha reta (SILVA e OLIVEIRA, 2006), diminuindo a disponibilidade desse recurso para as algas e prejudicando a fotossíntese. Taher (2013) também verificou tal problema na coloração do biodigestor suíno usado em seu trabalho, a turbidez do seu extrato concentrado prejudicou o crescimento de Scenedesmus sp.

No entanto, o extrato de Eichhornia puro apresentou crescimento satisfatório (principalmente Eic 40 e Eic 85). Sendo, portanto, uma opção econômica para a produção em larga escala, desde que seja ajustada sua diluição para proporcionar turbidez adequada.

Com relação aos volumes das células encontrados nos tratamentos, apesar de não apresentarem diferenças significativas $(p>0,05)$ que possam ser atribuídas a algum aspecto ambiental, é possível perceber uma tendência, entre as três diluições, de redução do volume celular. Isso pode ser explicado pelo fato de a turbidez crescente diminuir a penetração de luz na cultura, diminuindo a fotossíntese e a produção de compostos estruturais e fisiológicos responsáveis pelo crescimento celular. Claustre e Gostan (1987) documentaram em Hymenomonas elongata a relação entre os menores tamanho de células com a menor intensidade luminosa. O baixo volume de Eich + NPK registrado, pode ser explicado pela turbidez de 18,3 NTU desse tratamento em conjunto ao auto-sombreamento provocado pela elevada densidade encontrada.

Variações de tamanho e peso em algas como Scenedesmus são normais e demonstram uma plasticidade comum a algumas espécies (ROCHA et al., 2003; SIPAÚBATAVARES et al., 2011).

O valor do biovolume traduz a densidade do dia 20 e o volume das células. Dessa forma, quase todos os tratamentos apresentaram valores próximos, somente o Eic 130 apresentou valor muito menor e diferente dos demais, sendo semelhante ao Eic 85. Esses dois tratamentos apresentaram menor biovolume principalmente por apresentarem densidade celular muito pequena no dia 20 em comparação aos outros tratamentos. Eic NPK mesmo apresentando menor volume, obteve maior biovolume pelo fato da densidade algal no $20^{\circ}$ dia ter sido superior aos demais tratamentos, incluindo o controle.

\section{CONCLUSÕES}

Os dados obtidos permitem concluir que os tratamentos NPK, Pis + NPK e Eic + NPK apresentaram taxas de crescimento, densidade celular e biovolume compatíveis ao meio de cultura comercial (ASM1), em especial Eic + NPK que apresentou maior densidade celular e biovolume, representando assim alternativas aos meios de cultura convencionais e para 
utilização da biomassa dessa macrófita, proporcionando mais uma solução para os problemas ambientais associados a proliferação excessiva.

Dos tratamentos com extrato puro, Eic 40 apresentou resultados satisfatórios, podendo ser usado para o cultivo de $S$. acuminatus, desde que, observada a diluição ideal para evitar elevada turbidez do meio e consequente inibição do crescimento da alga.

Mais estudos devem ser realizados a fim de verificar a potencialidade do extrato puro de Pistia e investigar com mais detalhamento sua possível produção de compostos alelopáticos e as possíveis implicações ambientais dessa relação.

\section{AGRADECIMENTOS}

À CAPES pelo auxílio financeiro através da concessão da bolsa de mestrado.

\section{REFERÊNCIAS}

ALMEIDA, C.C.S. Avaliação do crescimento, padrões metabólicos e capacidade fotossintética de Microcystis panniformis Komárek et al. (Cyanobacteeria) e Ankistrodesmus gracilis (Reich) Korsikov (Chlorophyta) em fontes de nitrogênio inorgânicas e orgânicas. Ouro Preto, Brasil, p. 62, 2007. Dissertação - Programa de Pós-Graduação em Recursos Hídricos. UFOP.

ALMEIDA, S.Z.; FERNANDES, V.O. Effects of intensive fish-farming and domestic wastewater on the periphytic algal community in a tropical coastal lagoon (Juara, Brazil). Acta Scientiarum.

Biological Sciences. v. 35, p. 335-342, 2013. https://doi.org/10.4025/actascibiolsci.v35i3.17094

BARTMEYER, B.C.; OLIVEIRA, L.H.S.; COELHO, L.H.G. Comparação da retenção de cádmio e chumbo nos tecidos vegetais das macrófitas aquáticas Aquapé (Eichhornia sp) e Taboa (Typha sp) por biossorção e fitorremediação. HOLOS environment. v. 19, p. 145-159, 2019. http://dx.doi.org/10.14295/holos.v19i1.12295

BAUMGARTNER, T.R.S.; BURAK, J.A.M.; KOGIKOSKI, M.E. SEBASTIEN, N.Y.; ARROYO, P.A. Avaliação da produtividade da microalga Scenedesmus acuminatus (Lagerheim) Chodat em diferentes meios de cultivo. Revista Brasileira de Biociência, v. 11, p. 250-255, 2013.

CAMARGO, A.F.M.; PEZZATO, M.M.; HENRY-SILVA, G.G. Fatores limitantes à produção primária. In THOMAZ SM, BINI LM. ed. Ecologia e manejo de macrófitas aquáticas. Maringá: Eduem, $p$. 59-83, 2003.

CLAUSTRE, H.E.; GOSTAN, J. Adaptation of biochemical composition and cell size to irradiance in two microalgae: possible ecological implications. Marine Ecology Progress Series, v.40, p. 167174, 1987. https://doi.org/10.3354/meps040167

DALRYMPLE, O.K.; HALFHIDE, T.; UDOM, I.; GILLES, B.; WOLAN, J.; ZHANG, Q.; ERGAS, S. Wastewater use in algae production for generation of renewable resources: a review and preliminar results. Aquatic Biosystems, v. 9, p. 2, 2013. https://doi.org/10.1186/2046-9063-9-2 
DERNER, R.B.; OHSE, S.; VILLELA, M.; CARVALHO, S.M.; FETT, R. Microalgas, produtos e aplicações. Ciência Rural, v. 36, n. 6, p. 1959-1967, 2006. https://doi.org/10.1590/S0103$\underline{84782006000600050}$

DUARTE, I.D. Qualidade da água da lagoa Juara (Serra/ES): Avaliações físicas, químicas e respostas fisiológicas e citogenéticas em plantas. Vitória, Brasil. 102p., 2014. (Dissertação Programa de Pós-Graduação em Biologia Vegetal. UFES).

FERRELL, J.; SARISKY-REED, V. National algal biofuels technology roadmap. A technology roadmap resulting from the national algal biofuels workshop Washington U.S. Department of Energy, Office of Energy Efficiency and Renewable Energy, Biomass Program. 140p., 2010. Disponível: http://www1.eere.energy.gov/biomass/pdfs/algal biofuels roadmap.pdf. Acesso em: 18 fev. 2020.

FIORESI, T.B.; SIPAÚBA-TAVARES, L.H. Cultivo de Ankistrodesmus gracilis (Chlorophyta) em laboratório à base de esterco suíno. Revista Biotemas, v. 21, n. 1, p. 7-16, 2008. https://doi.org/10.5007/2175-7925.2008v21n1p7

GORHAM, P.R.; MCLACHLAN, J.; HAMMER, U.T.; KIM, W.K. Isolation and culture of toxic strains of Anabaena flos-aquae (Lyngb.). Bréb. Verh. Internat. Verein. Limnol., v. 15, p. 796-804, 1964. https://doi.org/10.1080/03680770.1962.11895606

GRECA, M.D.; FIORENTINO, A.; MONACO, P.; PREVITERA, L.; PINTO, G.; POLLIO, A. Release of potential allelochemical from aquatic plants. In MACIAS, F.A.; GALINDO, J.C.G.; MOLINILLO, J.M.G.; CUTLER, H.G. (Eds.) Recent advances in allelopathy. Cadiz, Serv. Pub. Univ. Cadiz, p. 255-262, 1999.

HENRY-SILVA, C.G.; CAMARGO, A.F.M. Valor nutritivo de macrófitas aquáticas flutuantes (Eichhornia crassipes, Pistia stratiotes e Salvinia molesta) utilizadas no tratamento de efluentes de aquicultura. Acta Scientiarum, v. 24, n. 2, p. 519-526, 2002.

HENRY-SILVA, C.G.; CAMARGO, A.F.M. Composição química de macrófitas aquáticas flutuantes utilizadas no tratamento de efluentes de aquicultura. Planta Daninha, v. 24, n. 1, p. 21-28, 2006. https://doi.org/10.1590/S0100-83582006000100003

KRUSE, O.; HANKAMER, B. Microalgal hydrogen production. Current Opinion in Biotechnology, v. 21, n. 3, p. 238-243, 2010. https://doi.org/10.1016/j.copbio.2010.03.012

LEMES, J.L.V.B.; SCHIRMER, W.N.; CALDEIRA, M.V.W.; KAICK, T.V.; BÁRBARA, R.R. Tratamento de esgoto por meio de zona de raízes em comunidade rural. Revista Acadêmica: Ciências Agrárias e Ambientais, v. 6, n. 2, p. 169-179, 2008.

LIMA, B.A.A.; LIBORIO, M.P.; HADAD, R.M. Análise espaço-temporal do crescimento de macrófitas e sua aplicação no monitoramento da qualidade da água. Ra'ega o espaço geográfico em análise, v. 45, n. 01, p. 45-57, 2018. http://dx.doi.org/10.5380/raega.v45i1.50300

LIMA, M.R.; TAFFAREL, A.D.; REISSMANN, C.B.; CRUZ, A.C.L.; DEPINÉ, H. Avaliação do crescimento e retenção de nutrientes provenientes da eutrofização, em três macrófitas aquáticas. In: Congresso brasileiro de ciência do solo, Ribeirão Preto, 2003. Anais. Ribeirão Preto: UNESP, SBCS, 2003a. CD-Rom.

LOURENÇO, S.O. Cultivo de microalgas marinhas - Princípios e aplicações. Rima, São Carlos, São Paulo, 588p., 2006. 
MARTINS, D.; COSTA, N.V.; TERRA, M.A.; MARCHI, S.R.; VELINI, E.D. Caracterização química das plantas aquáticas coletadas no reservatório de Salto Grande (Americana - SP). Planta Daninha, v. 21, p. 21-25, 2003. https://doi.org/10.1590/S0100-83582003000400004

MILITÃO, F.P.; FERNANDES, V.O.; BASTOS, K.V.; COLEPICOLO, P.; MARTINS, A.P.; MACHADO, L.P. Nutritional value changes in response to temperature, microalgae mono and mixed cultures. Acta Limnologica Brasiliensia, v. 31, p.1-12, 2019. FapUNIFESP (SciELO). http://dx.doi.org/10.1590/s2179-975x7118

NDIMELE, P.E.; KUMOLU-JOHNSON, C.A.; ANETAKHAI, M.A. The invasive aquatic macrophyte, Water Hyacinth (Eichhornia crassipes (Mart.) Solm-Laubach: Pontedericeae: Problems and prospects. Research Journal of Environmental Sciences, v. 5, n. 6, p. 509-520, 2011. https://doi.org/10.3923/rjes.2011.509.520

PIÑA, P.; MEDINA, M.A.; NIEVES, M.; LEAL, S.; LÓPEZ-ELIAS, J.A.; GUERRERO, M.A. Cultivo de quatro espécies de microalgas com diferentes fertilizantes utilizados em aquicultura. Ver. Invest. Mar., v. 28, n. 3, p. 225-236, 2007.

PRABAKARAN, P.; RAVINDRAN, D. Scenedesmus as a potential source of biodiesel among selected microalgae. Current Science, v. 102, n. 4, p. 606-620, 2012.

ROCHA, M.S.J.; GARCIA, J.E.C.; HENRIQUES, M.H.F. Growth aspects of the marine microalga Nannochloiropsis gaditana. Biomolecular Engineering, v. 20, n. 4-6, p. 237-242, 2003. https://doi.org/10.1016/S1389-0344(03)00061-3

SAMPAIO, E.V.S.B.; OLIVEIRA, N.M.B. Aproveitamento da macrófita aquática Egeria densa como adubo orgânico. Planta Daninha, v. 23, n. 2, p. 169-174, 2005. https://doi.org/10.1590/S0100$\underline{83582005000200001}$

SILVA, S.A.; OLIVEIRA, R.O. Manual de análises físico-químicas de águas de abastecimento e residuárias. ABES, Campina Grande: Paraíba. 266p., 2006.

SILVA, A.L.M.; SILVA, T.T.S.; GONÇALVES, E.A.P.; SANTOS, S.M. Uso sustentável de macrófitas no tratamento de efluentes: uma revisão sistemática. Journal of Environmental Analysis and Progress, v. 04, n. 04, p. 228-238, 2019. https://doi.org/10.24221/jeap.4.4.2019.2590.228-238

SIPAÚBA-TAVARES, L.H.; ROCHA, O. Cultivo em larga escala de organismos planctônicos para alimentação de larvas e alevinos de peixes: I - Algas Clorofíceas. Biotemas, v. 6, n.1, 93-106, 1993.

SIPAÚBA-TAVARES, L.H.; BRAGA, F.M.S. The feeding activity of Colossoma macropomum larvae (tambaqui) in fishponds with water hyacinth (Eichhornia crassipes) fertilizer. Brazilian Journal of Biology, v. 67, n. 3, p. 459-466, 2007. https://doi.org/10.1590/S1519-69842007000300010

SIPAÚBA-TAVARES, L.H.; IBARRA, L.C.C.; FIORESI, T.B. Cultivo de Ankistrodesmus gracilis (Reisch) Korsikov (Chlorophyta) em laboratório utilizando meio $\mathrm{CHU}_{12}$ e de macrófita com NPK. Boletim do Instituto de Pesca, São Paulo, v. 35, n. 1, p. 111-118, 2009.

SIPAÚBA-TAVARES, L.H.; MILLAN, R.N.; BERCHIELLI, F.A.; BRAGA, F.M.S. Use of alternative media and different types of recipients in a laboratory culture of Ankistrodesmus gracilis (Reinsch) Korshikov (Chlorophyceae). Acta Scientiarum. Biological Sciences, v. 33, n. 3, p. 247-253, 2011. https://doi.org/10.4025/actascibiolsci.v33i3.8046

SIPAÚBA-TAVARES, L.H.; PEREIRA, A.M.L. Large scale laboratory cultures of Ankistrodesmus gracilis (Reisch) Korsikov (Chlorophyta) and Diaphanosoma biergei Korinek, 1981 (Cladocera). 
Brazilian Journal of Biology, v. 68, n. 4, p. 875-883, 2008. https://doi.org/10.1590/S1519$\underline{69842008000400025}$

STEIN, J.R. Handbook of Phycological Methods: Culture Methods and Growth measurements.

Cambridge University Press, Cambridge, 472p., 1973.

TAHER, D.M. Biodiesel de microalgas cultivadas em dejeto suíno biodigerido. Curitiba, PR. 102p., 2013 (Dissertação - Engenharia e Ciência dos Materiais, Setor de Tecnologia, da UFPR).

TEPE, Y.; NAZ, M.; TÜRKMEN, M. Utilization of different nitrogen sources by cultures of Scenedesmus acuminatus. Turkish Journal of Fisheries and Aquatic Sciences, v. 6, p. 123-127, 2006.

TRAINOR, F.R. Biological aspects of Scenedesmus (Chlorophyceae)-phenotypic plasticity. Nova Hedwigia, Beiheft. 117p., 1998.

YIN, K.; HARRISON, P.J.; DORTCH, Q. Lack of ammonium inhibition of nitrate uptake for a diatom grown under low light conditions. Journal of Experimental Marine Biology and Ecology, v. 228, p. 151-165, 1998. https://doi.org/10.1016/S0022-0981(98)00025-2 